\title{
MOSA (Mission-Oriented Sensor Arrays): uma nova abordagem para o sensoriamento remoto embarcado em VANT's
}

\author{
Nina Machado Figueira* \\ Onofre Trindade Junior* \\ Eduardo do Valle Simões* \\ Ana Carolina de Sousa Silva**
}

Recebido el 28 de abril de 2015; aceito el 16 de agosto de 2015

\begin{abstract}
The use of Unmanned Autonomous Vehicles (UAV's) is progressively shifting from military missions to civilian applications and becoming a viable alternative for remote sensing and automated thematic mapping. This work presents the concept of Mission-Oriented Sensor Arrays, or MOSA. A MOSA system is composed of a set of sensors and computing devices that are integrated and embedded into an UAV to perform specific missions autonomously, among them, mapping missions. The main objective of a MOSA system is the on-board processing of sensory data, producing real-time, ready-to-use information. MOSA also allows the reduction of information traffic on the often limited communication channel between the aircraft and the ground station. Within the MOSA concept, the aircraft is structured as such: mission control (MOSA) and flight control (autopilot). This increases the robustness of the whole system, since it is only necessary to exchange the MOSA to adapt the UAV to a new mission. In this paper we present a reference architecture and a development methodology for MOSA systems. The specification of a MOSA system applied to the mapping of ground-based sound sources is also presented to illustrate the proposed approach. The modeled MOSA is based on the integration of

* Instituto de Ciências Matemáticas e de Computação (ICMC) da Universidade de São Paulo, Campus São Carlos, curso de pós-graduação em Ciência da Computação, Av. Trabalhador São-Carlense, 400, Centro, São Carlos, SP; e-mail: nina.figueira@dsg.eb.mil.br; onofre.trindade.junior@gmail.com; simoes@icmc.usp.br

** Faculdade de Zootecnia e Engenharia de Alimentos (FZEA) da Universidade de São Paulo, Campus Pirassununga, Rua Duque de Caxias Norte, 225, Vila Esperança, Pirassununga, SP, e-mail: anacss@usp.br
\end{abstract}


data from a thermal imaging sensor and a tridimensional sensor for the localization of sound sources. The positional information provided by the applied sensors are integrated into a single cartographic base. This article aims the presentation of MOSA systems concept, including architecture, design methodology, and a reference application model for the automatic mapping of sound sources.

Key words: Unmanned Aerial Vehicles (UAV), Mission-Oriented Sensor Arrays (MOSA), automated thematic mapping, embedded processing, sensor data fusion.

\section{Resumen}

El uso de Vehículos Aéreos No Tripulados (UAV, por sus siglas en inglés) ha emigrado gradualmente de la esfera militar al empleo civil, siendo una alternativa viable, versátil en numerosas actividades relacionadas con la teledetección, en particular la relacionada con la generación automática de mapas temáticos. En este trabajo se describe el concepto de matrices de sensores orientados a misión (MOSA - Mission-Oriented Sensor Arrays). Un sistema de MOSA comprende un conjunto de sensores y dispositivos computacionales integrados que pueden ser transportados en un UAV para la realización de misiones específicas autónomas, incluidas las misiones de mapeo. El objetivo principal del sistema MOSA es el procesamiento a bordo de los datos sensoriales, la producción en tiempo real y la información lista para su uso. MOSA también permite la reducción del tráfico de información en el canal de comunicación, a menudo limitada entre la aeronave y la estación terrestre. Dentro del concepto MOSA, el avión se estructura como control de la misión (MOSA) y control de vuelo (piloto automático). Esto aumenta la robustez de todo el sistema, ya que únicamente es necesario un cambio del MOSA para adaptar el UAV a una nueva misión. Así mismo se presenta una arquitectura de referencia y una metodología de desarrollo de sistemas de MOSA. La especificación de un sistema de MOSA aplicada a la cartografía de las fuentes de sonido basados en tierra también se presenta para ilustrar el enfoque propuesto. El MOSA modelado se basa en la integración de los datos de un sensor de imagen térmica y un sensor tridimensional para la localización de las fuentes de sonido. Las informaciones de posición proporcionada por los sensores aplicados están integradas en una sola base cartográfica. Este artículo tiene como objetivo la presentación de MOSA como un concepto de sistemas, incluyendo la arquitectura, la metodología de diseño, y un modelo de aplicación de referencia para la asignación automática de las fuentes de sonido.

Palabras clave: Vehículos Aéreos No Tripulados, Generación Automática de Mapas Temáticos, matrices de sensores orientados a misión, procesamiento a bordo, integración de datos. 


\section{Resumo}

O uso de Veículos Aéreos Não Tripulados (VANT's), têm migrado rapidamente da esfera militar para o emprego civil, mostrando ser uma alternativa viável versátil em inúmeras atividades relacionadas com o sensoriamento remoto, particularmente às relacionadas com a geração automática de mapas temáticos. Em paralelo está em desenvolvimento o conceito de arranjos de sensores orientados à missão, MOSA (Mission-Oriented Sensor Array), que é objeto deste artigo. Um sistema MOSA é composto por um conjunto de sensores e dispositivos computacionais integrados que podem ser embarcados em VANT's, visando à realização de missões específicas de forma autônoma, entre as quais, missões de mapeamento. O objetivo mais importante de um sistema MOSA é o processamento a bordo da aeronave dos dados básicos obtidos pelos sensores, produzindo-se em tempo real informação pronta para uso. Sistemas MOSA também permitem a redução do volume de informação que trafega no canal de comunicação existente entre a aeronave e a estação de solo, normalmente com capacidade de comunicação limitada. A aeronave, sob o ponto de vista do conceito MOSA é estruturada em: controle de missão (MOSA) e o controle de voo (piloto automático). Isso aumenta a segurança de todo o sistema, uma vez que, para adaptar o VANT a uma nova missão, basta substituir o sistema MOSA existente. Neste trabalho, é apresentada uma arquitetura de referência de sistemas MOSA e uma metodologia de desenvolvimento para esta classe de sistemas. É descrita a especificação de um sistema MOSA para uso no mapeamento de fontes sonoras em solo para esclarecer detalhes da abordagem proposta. O MOSA modelado nesta aplicação é baseado na integração de dados provenientes de um sensor de imageamento termal e um sensor tridimensional para localização de fontes sonoras. Um dos aspectos importantes é a integração das informações de posicionamento providas pelos sensores utilizados em uma base cartográfica única. Este trabalho foca na apresentação do conceito de sistemas MOSA, incluindo arquitetura, metodologia de desenvolvimento e a modelagem de uma aplicação de referência, representada pelo sistema de mapeamento automático de fontes sonoras.

Palavras chave: Veículos Aéreos Não Tripulados (VANT's), Mission-Oriented Sensors Arrays (MOSA), Mapeamento temático automatizado, Processamento embarcado, fusão de dados de sensores.

\section{Introdução}

O sensoriamento remoto é a arte, a ciência e a tecnologia empregadas para obter, sem contato físico, informações confiáveis sobre o ambiente e os objetos físicos presentes, através do registro, interpretação de imagens e representações digitais dos padrões de energia obtidos por sistemas sensores. A fotogrametria é a forma de 
sensoriamento remoto mais antiga, sendo que usa principalmente imagens aéreas obtidas de aeronaves para fins de geração de mapeamento topográfico, com base em um arcabouço próprio para medições tridimensionais, embora antes do advento das imagens óticas de satélites, tivesse também como atividade fim a geração de mapas temáticos, através da fotointerpretação.

A relação estabelecida entre o sensoriamento remoto e o processo de representação gráfica é iniciada no momento da aquisição de imagens da superfície, compondo a base que possibilita a construção de mapas e produtos cartográficos em geral. As diversas formas de mapeamento tais como ortofotos, mosaicos, cartas topográficas, mapas temáticos, modelos digitais de elevação, entre outras, são fundamentais para o planejamento e controle de atividades civis e militares.

O termo UAV (Unmanned Aerial Vehicle) foi adotado pela FAA (Federal Aviation Administration) e pela comunidade acadêmica internacional para designar sistemas que incluem não apenas os aviões, mas todos os elementos associados, tais como a carga útil (payload), a estação de controle terrestre (Ground Control Station - GCS) e os links de comunicação (GAO, 2008). No Brasil esses sistemas são conhecidos como VANT's, ARP's (Aeronaves Remotamente Pilotadas) ou vulgarmente denominados Drones. Um VANT pode operar por um longo período de tempo sem a intervenção de um operador humano. Os VANT's têm sido amplamente utilizados como plataformas nas quais são embarcados sensores de altitude, vídeo e imagens, caracterizando uma forma relevantede aquisição de informações geográficas.

Existem diferentes tipos de VANT's apresentando diferentes capacidades. Algumas aeronaves podem voar de forma autônoma, seguindo uma trajetória de voo pré-programada (baseada em um grid ou uma sequência de way points) (Trindade et al., 2010), enquanto outras voam recebendo comandos de pilotos humanos a partir da GCS. De acordo com a Agência Nacional de Aviação Civil (ANAC) atualmente, por questões de segurança do tráfego aéreo, não é permitido realizar voos autônomos além da linha de visada com a GCS. O piloto na GCS deve estar em condições de assumir o voo manual no caso de haver incidentes que comprometam a segurança da operação, na maioria dos casos o processamento dos dados é realizado em escritório. Já no cenário militar as GCS's (além de controlar a missão) são dotadas de sistemas de inteligência e auxílio à tomada de decisão que são capazes de processar dados recebidos e gerar quadros situacionais inteligentes em tempo real. O tamanho da aeronave pode variar bastante e a GCS pode ser implementada em smartphones, tablets, notebooks ou redes de estações de controle em solo. A aeronave pode variar não apenas em tamanho, mas também na forma, no tipo de propulsão e no desempenho.

Muitos artigos relacionados a VANT's, encontrados na literatura, apresentam sistemas que utilizam abordagens tradicionais (OSD, 2002; OSD, 2005; OSD, 
2009a; OSD, 2009b, Valavanis, 2007, Trindade et al., 2002). Outros documentos procuram prever os avanços esperados para os VANT's nos próximos anos e são publicados periodicamente por organizações militares, como a Força Aérea dos Estados Unidos (USAF, 2009).

Os VANT's devem popular nos próximos anos uma boa parte do espaço aéreo para a realização de diferentes missões civis, desde o monitoramento agrícola e ambiental, passando por inspeções de fronteira e defesa pública até o transporte automático de cargas.Esse uso crescente dos VANT's tem contribuído fortemente para a sua popularização, passando a ser comercializados de forma mais ampla.

A geração de informações processadas, a partir dos dados brutos coletados pelos sensores, é a principal tarefa em aplicações civis. Este processamento geralmente é realizado no escritório, por pessoal treinado utilizando algoritmos específicos para a o mapeamento. Na maioria dos casos existe inspeção manual dos dados, mas nem sempre é possível alcançar os requisitos de resolução temporal, em virtude dos sistemas não contemplarem o processamento de dados em tempo real para a aplicação desejada. Um bom exemplo é o processamento de dados para aplicativos de gerenciamento agrícola, onde a detecção de doenças e pragas deve gerar mapas temáticos e de aplicativos dentro de um intervalo de tempo específico, caso contrário, a cultura pode ser seriamente comprometida. Vale a pena notar que o ciclo de vida de culturas anuais, como a soja, se estende por apenas três meses a cada ano e qualquer ação corretiva deve ser feita poucos dias após a coleta de dados.

A arquitetura MOSA proposta neste trabalho tem o potencial de fornecer informações processadas para utilização imediata (pronto emprego), reduzindo a necessidade de mão de obra especializada na ECS. Além disso, essa arquitetura também visa reduzir ou eliminar as exigências sobre os canais de comunicação utilizados para o transporte de dados em tempo real entre a aeronave e a ECS, tais como imagens de alta resolução. No exemplo da agricultura de precisão, mapas temáticos requerem muito menos memória e consequente banda do canal de comunicação do que os dados sensoriais (normalmente imagens), utilizados na sua geração.

A geração automática de mapas, a partir de dados adquiridos por arranjos de sensores MOSA, exige a utilização de conhecimentos provenientes de diversas disciplinas notadamente os Sistemas de Computação e fotogrametria. Neste trabalho, apresenta-se uma proposta de como integrar essas áreas, dentro da arquitetura proposta para os sistemas MOSA. São apresentadas a arquitetura MOSA e a definição dos principais aspectos do sistema através de um modelo e uma implementação de referência. As técnicas propostas neste trabalho facilitarão o desenvolvimento de aplicações automatizadas de VANT's, permitindo que esses veículos possam ser inseridos e incorporados mais facilmente no espaço aéreo e 
contribuindo para a sua disseminação. Nesse sentido serão apresentados nas seções seguintes: uma seleção de trabalhos relacionados com a pesquisa em questão; uma introdução ao modelo e à arquitetura MOSA; a metodologia de desenvolvimento de um MOSA; uma proposta de modelagem para um MOSA no contexto do monitoramento ambiental; as conclusões e os trabalhos futuros.

\section{Trabalhos relacionados}

Diversos trabalhos têm utilizado sensores embarcados em VANT's para a produção de mapas e outras formas de representação de informações prontas para o usuário ou aplicação fim. Muitas dessas incorporam etapas de processamento automático ou semiautomático (supervisionado), feito a bordo da aeronave, como proposto em etapas que compõe a construção de sistemas MOSA. Essas iniciativas, entretanto, não contemplam uma arquitetura ou uma organização geral de hardware e software para facilitar a implementação de novas aplicações.

A quantidade de trabalhos relacionados que pode ser encontrada é bastante grande, refletindo o interesse cada vez maior no emprego de VANT's em aplicações civis. Nos parágrafos abaixo menciona-se alguns destes trabalhos, considerados representativos, com uma breve descrição dos seus objetivos e resultados obtidos. Nenhum dos referidos trabalhos pode ser considerado um MOSA, no entanto servem como referência para alguns dos blocos que compõe a totalidade do sistema.

\section{Fusão de dados de multissensores}

Sistemas multissensores vem sendo utilizados tanto para mapeamento de ambientes (Abielmona et al., 2010) como para detecção e classificação de atividades humanas (Ugolotti et al., 2013). Turolla et al. (2004) desenvolveram um método para o rastreamento de objetos utilizando múltiplas câmeras de vídeo em aplicações de vigilância utilizando fusão de dados. Li Ying-cheng et al. (2011) utilizaram fotografias aéreas adquiridas por um VANT para realizar o mapeamento topográfico de uma ilha.

Nagai et al. (2009) desenvolveram um sistema de mapeamento tridimensional a partir da integração de multissensores embarcados em VANT's. Polychronopoulos et al. (2006) apresentaram um trabalho sobre fusão de dados em plataformas multissensores para a percepção de áreas utilizando um algoritmo de fusão que oferece novas soluções para o problema do gerenciamento de espaços e percepção de largura. Molina et al. (2012) e (2013) discutiram os desafios técnicos e operacionais do uso combinado de visão térmica e óptica (infravermelho e visível) para o reconhecimento de pessoas. Com base na análise da qualidade geodésica e no uso do sistema EGNO's (European Geostationary Navigation Overlay Service) foi possível obter uma estimativa realista e precisa da posição de pontos de 
interesse no terreno. Foi desenvolvida uma plataforma computacional reconfigurável visando aplicações de baixo custo.

Klausner et al. (2006) demonstraram o potencial de combinar fusão multissensorial à computação embarcada em tempo real. No que se refere ao processamento embarcado de imagens tem-se desafios como complexidade dos algoritmos, o volume dos dados processados e limitações para a transmissão desses dados. Um sistema de processamento embarcado de imagens deve conciliar uma solução para os problemas mencionados com um baixo consumo de energia. Mejías et al. (2007) fez um levantamento do estado da arte em processamento embarcado de imagens.

\section{Processamentoembarcado de dados}

Grigorius, Dollas e Bourbakis (2012) apresentaram uma arquitetura de software reconfigurável para a segmentação de cores em sistemas embarcados utilizando o método FRS (Fuzzy Like Reasoning). O método foi escolhido devido ao bom desempenho em aplicações envolvendo: deteç̧ão de face, deteç̧ão de movimento e reconhecimento automático de alvos.

Kealy et al. (2013) apresentam um estudo sobre navegação cooperativa (ou colaborativa) utilizando diferentes plataformas de sensores de posicionamento e de localização com precisão diferentes para o compartilhamento de localizações absolutas e relativas. Esse é um cenário típico de aplicação para: deslocamento de tropas no terreno, enxames de VANT's, equipes de robôs e equipes de emergência.

\section{Mapeamento móvel}

Yi Lin et al. (2013) propõem um estudo no qual apresentam o desenvolvimento de um novo sistema para o Sensoriamento Remoto, o AGRS (Air-Ground Remote Sensing), visando o levantamento de pontos de interesse. O trabalho consiste basicamente em associar o AGRS a imagens de um sistema de mapeamento móvel (Mobile Mapping System - MMS) a bordo de um VANT.

Hruska et al. (2005) apresentam um fluxo de trabalho/arquitetura para o processo de aquisição de imagens georreferenciadas de alta resolução utilizando pequenos VANT's, incluindo o planejamento inicial da missão, a seleção e a integração dos sensores, a coleta, o processamento e a análise das imagens visando a obtenção de informações geográficas para o mapeamento topográfico.

Zhang e Kovacs (2012) realizaram uma revisão de trabalhos envolvendo a utilização de pequenos VANT's na agricultura de precisão. Os resultados destes estudos indicam que, para proporcionar um produto final confiável para os agricultores, são necessários: avanços no projeto das plataformas de aquisição, análise de detalhes técnicos da produção, padronização no georreferenciamento e mosaicagem de imagens. 
Choi e Lee (2013) desenvolveram um algoritmo de triangulação aérea sequencial para o georreferenciamento direto em tempo real de sequências de imagens adquiridas por um sistema multissensor aerotransportado. Este algoritmo pode ser utilizado para aplicações que requerem georreferenciamento de imagens em tempo real, tais como no monitoramento de desastres e na navegação baseada em imagens.

Li-Chee-Ming e Armenakis (2012) introduziram um sistema de mapeamento estéreo móvel (MSMS - Mobile StereoMapping System), utilizando VANT's, projetado para a navegação rápida e coleta de dados espaciais tridimensionais por meio de georreferenciamento direto e integração de multissensores.

\section{Sistemas MOSA - Conceitos e Arquitetura}

Arquitetura é uma estrutura que identifica, define e organiza componentes (Shaw e Garlan, 1996). O relacionamento e os princípios de projeto dos componentes, funções e interface estabelecidas entre os subsistemas também podem ser definidos por uma arquitetura. Um modelo de referência para uma arquitetura é uma estrutura onde as entidades, relacionamentos e unidades de informação envolvidos nas interações entre e dentro dos subsistemas e componentes são definidos e modelados. Em resumo, é um modelo que incorpora o objetivo básico ou a ideia do sistema e pode ser considerado como uma referência para várias finalidades (Shaw e Garlan, 1996).

A Figura 1 mostra a proposta de diagrama funcional simplificado da arquitetura MOSA e o inter-relacionamento entre os componentes do sistema. O diagrama pode variar em complexidade e número de componentes, dependendo de cada aplicação em particular.

Diversos sensores podem integrar um sistema MOSA. Os dados provenientes desses sensores inicialmente são adquiridos e pré-processados por módulos específicos de hardware e/ou software. Estes dados podem sofrer processamento adicional em módulos especializados que são utilizados na geração de informações úteis como saída do sistema.

Fontes de dados internas podem estar presentes em sistemas MOSA. Entre essas, citam-se os dados provenientes de unidades inerciais, dados de posicionamento provenientes de receptores GPS e dados previamente armazenados em uma base de dados local, como por exemplo, modelos digitais de elevação do terreno (DTM —Digital Terrain Model).

Todos os dados disponíveis podem ser combinados no módulo de "Fusão de Dados e Controle da Missão", gerando resultados finais, p. ex., coordenadas de elementos de interesse para a construção de mapas temáticos, que podem ser armazenados na base de dados local e transmitidos para a estação de controle de terra através de serviços de comunicação providos pela aeronave. 


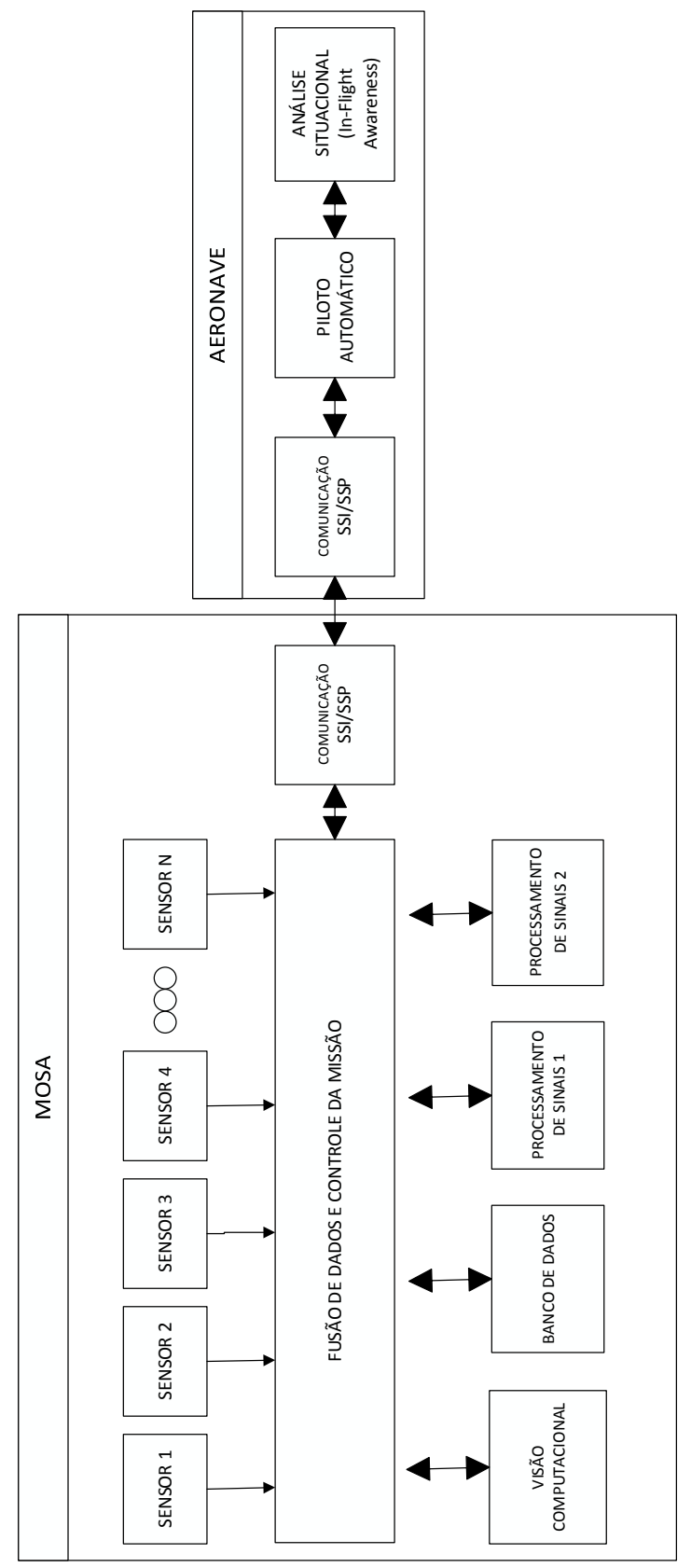

O) 


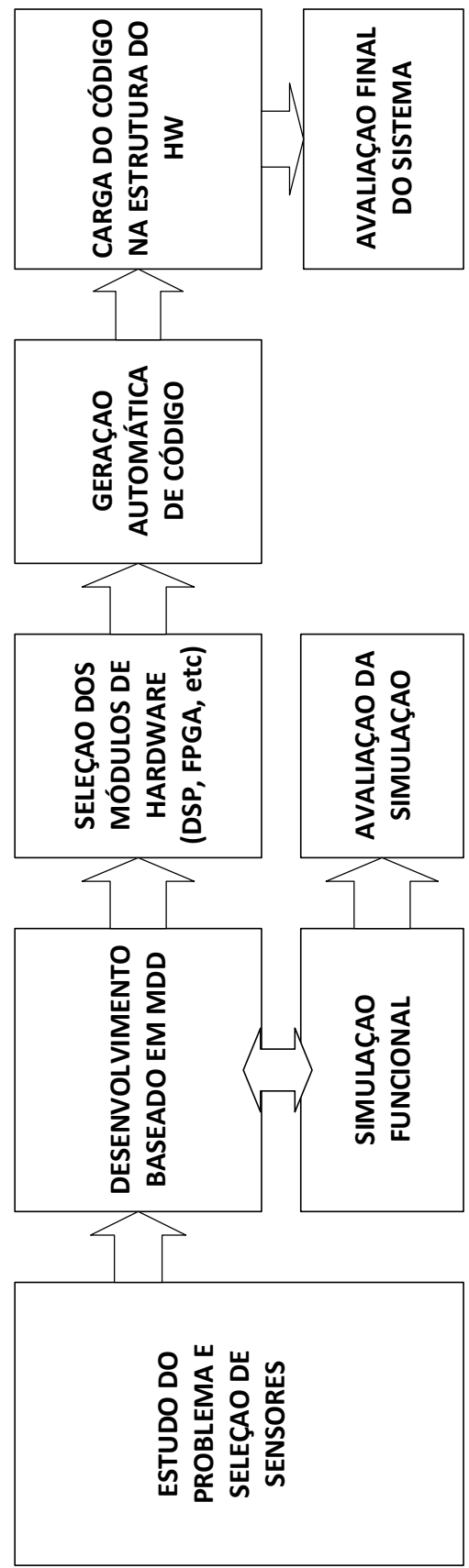


Para comunicar-se com a aeronave, o MOSA utiliza uma interface padrão, denominada SSP/SSI (Smart Sensor Protocol/Smart Sensor Intertace) SSP é o protocolo de comunicação utilizado enquanto SSI é a interface que permite que o sistema MOSA possa utilizar diversos serviços providos pela aeronave, notadamente os serviços de transporte (voo) e de comunicação com a estação de controle em solo. A Figura 1 ilustra este relacionamento.

Conceitualmente é possível utilizar sistemas MOSA em diferentes aeronaves, o que depende apenas das interligações de tecnologias em uma arquitetura flexível. $\mathrm{O}$ protocolo deve utilizar mecanismos plug-and-play para determinar se a aeronave é ou não capaz de realizar uma determinada missão. Por exemplo, em uma missão específica pode ser necessária autonomia muito grande ou maior estabilidade de voo, entre outros fatores limitantes. O sistema MOSA deve ser capaz de determinar se a missão prevista pode ou não ser realizada, de forma plena ou parcial.

\section{Materiais e Métodos para o desenvolvimento de um MOSA}

O MOSA integra vários sensores, módulos de hardware/software e hardware de comunicação. Seus principais componentes são:

- Hardware: sistema modular e configurável dispondo de sensores específicos para a missão e diferentes níveis de processamento de sinais/dados, dependendo da missão pretendida. Pode incluir componentes de hardware programável baseado em FPGA (Field Programmable Gate Arrays);

- Software: Componentes de software desenvolvidos com metodologia MDD (Model Driven Development). Embora existam diversos ambientes de desenvolvimento baseados em MDD, o Matlab Simulink ${ }^{\circledR}$ (MathWorks Inc., R2013b) tem sido utilizado nas primeiras aplicações. As principais bibliotecas utilizadas são a de processamento de sinais e imagens, visão computacional e mapeamento, além de blocos básicos, com capacidade de geração automática de código.

A configuração de hardware e software de um sistema MOSA varia de acordo com a necessidade de cada missão. Para desenvolver uma nova aplicação, é proposta a sequência de etapas ilustrada na Figura 2.

A metodologia de desenvolvimento segue a Prolices, definida em Braga et al. (2011). Recomenda-se, para melhor entendimento do contexto de desenvolvimento a leitura desta referência citada. As etapas do fluxo de desenvolvimento apresentadas na Figura 2 são descritas nos itens seguintes:

I. Estudo do problema e seleção de sensores: nessa etapa é realizada a especificação e projeto do sistema MOSA compreendendo o levantamento de 
requisitos, seleção e verificação das características de cada tipo de sensor a ser utilizado, o plano de voo, busca por documentos cartográficos além de informações político-sociais referentes à área de interesse.

Entradas:

- Estudo do terreno (onde será executada a missão?);

- Requisitos da missão;

- Especificações técnicas da aeronave;

- Especificação de múltiplos sensores (sonares, radares, visíveis, lasers);

- Normas de operação;

- Manuais, avisos, documentos cartográficos;

- Previsões climáticas;

- Natureza dos sinais e dos alvos,

- Resoluções espaciais, radiométrica e temporal da aquisiçao dos alvos;

- Escala do mapa temático;

- Finalidade de missão.

Saídas:

- Definição dos sensores;

- Plano de voo;

- Especificações da missão (alvos, tipo de mapa temático a ser produzido, resolução temporal da aquisição);

- Métrica utilizada para avaliar o sistema.

II. Desenvolvimento do sistema baseado em Model Driven Development (MDD): no desenvolvimento orientado a modelos, um DFD (Diagrama de Fluxo de Dados) é construído onde são definidos inicialmente, com base nos requisitos da missão, os blocos de processos que serão utilizados na solução do problema. Em seguida é feita a decomposição dos blocos, de forma hierárquica e incremental, até o nível das funções básicas necessárias à execução de cada bloco. As funções básicas são selecionadas dentro da taxonomia de funções básicas desenvolvida para sistemas MOSA (Braga et al., 2011). Alguns dos blocos podem ser especificados utilizando-se máquinas de estados finitos, que facilitam o projeto de blocos com comportamento reativo ou de tempo real. Alguns blocos podem ser especificados em linguagem Matlab, diretamente, quando isso for conveniente. É possível também o encapsulamento de código desenvolvimento em outra linguagem e outras metodologias.

Entradas:

- Especificações da missão e do voo.

Saídas:

- Modelos;

- Fluxogramas;

- Estado de máquinas finitas; 
- Taxonomias de blocos defunções.

III. Simulação funcional: essa etapa tem por objetivo validar funcionalmente o sistema através da simulação do mesmo no ambiente Simulink.

Entradas:

- Modelos;

- Fluxogramas;

- Estado de máquinas finitas;

- Taxonomias de blocos de funções.

Saídas:

- Modelo Simulink;

- Requisitos de HW.

IV. Seleção dos módulos de hardware: além da verificação funcional do sistema, a simulação pode fornecer dados de desempenho que podem nortear a partição hardware/software e podem servir de apoio para a seleção da potência computacional dos módulos de processamento utilizados. Neste sentido, blocos funcionais podem ser mapeados em processos dentro de módulos de processamento enquanto sistemas que demandam alta potência computacional podem ser mapeados em módulos de hardware específicos, eventualmente implementados utilizando-se FPGA's (Field Programmable Gate Arrays);

Entradas:

- Modelo Simulink;

- Requisitos de HW.

Saídas:

- Seleção dos HW.

V. Geração automática de código: os algoritmos especificados pelo DFD do sistema e as outras técnicas de especificação mencionadas podem dar origem, de forma automática, aos códigos que serão executados nos módulos de processamento. Módulos de hardware também podem ser gerados automaticamente por este procedimento, utilizando-se código gerado em Linguagens de Descrição de Hardware tal como VHDL e Bluespec;

Entradas:

- Modelos Simulink;

- Seleção dos HW.

Saídas:

- Código gerado automaticamente.

VI. Carga do código na estrutura do hardware: uma vez carregado no hardware, o código pode ser avaliado utilizando-se a técnica de hardware-in-the-loop (Braga et al., 2011). Essa técnica permite a avaliação do sistema em solo, evitando-se os riscos associados com os testes em voo. O ambiente Matlab/Simulink pode 
ser utilizado nesta etapa para a geração/simulação das entradas e saídas correspondentes aos sensores e ao protocolo SSP;

Entradas:

- Código gerado automaticamente.

Saídas:

- Hardware implementado com o código proposto.

VII.Avaliação final do sistema: por ser tratar de um sistema embarcado, os testes finais de aceitação do sistema devem ser realizados nas condições reais de utilização, neste caso específico, com a realização de missões aéreas especificamente planejadas.

Entradas:

- Protótipo do sistema,

- Estudo de caso;

- Dados de referência.

Saídas:

- PSNR (Peak Signal-to-Noise Ratio);

- $\quad$ PEC (Padrão de Exatidão Cartográfica);

- RMSE (Root-Mean-Square Error).

\section{Exemplo de Sistema MOSA - Mapeador Automático de Fontes Sonoras}

A atividade de monitoramento ambiental consiste na realização de medições e/ou observações específicas, dirigidas a indicadores e parâmetros, com a finalidade de verificar se determinados impactos estão ocorrendo, podendo ser dimensionada sua magnitude e avaliada a eficiência na adoção de eventuais medidas preventivas e corretivas (Bitar e Ortega, 1998). Segundo Machado (1995), a elaboração de um registro dos resultados do monitoramento ambiental (uso do solo, análise do comportamento animal, catástrofes, etc) é de fundamental importância para o acompanhamento da situação e auxilio a tomada de decisão em todas as esferas de nossa sociedade.

Muitas são as atividades de monitoramento ambiental que atualmente demandam soluções diferenciadas que vão além da aquisição de imagens. No sentido de complementar e enriquecer as informações fornecidas pelas câmeras convencionais (fotográfica e vídeo) é proposta a utilização de sensores acústicos.

O exemplo de MOSA apresentado nessa seção integra o processamento de sons, imagens, coordenadas, ângulos de atitude das plataformas de aquisição e dados de referência visando à identificação e a classificação automática de alvos. A Figura 3 mostra um diagrama de blocos da proposta de um sistema para o mapeamento automático de fontes sonoras em solo no contexto do monitoramento ambiental (detecção de caçadores e atividades ilícitas). 


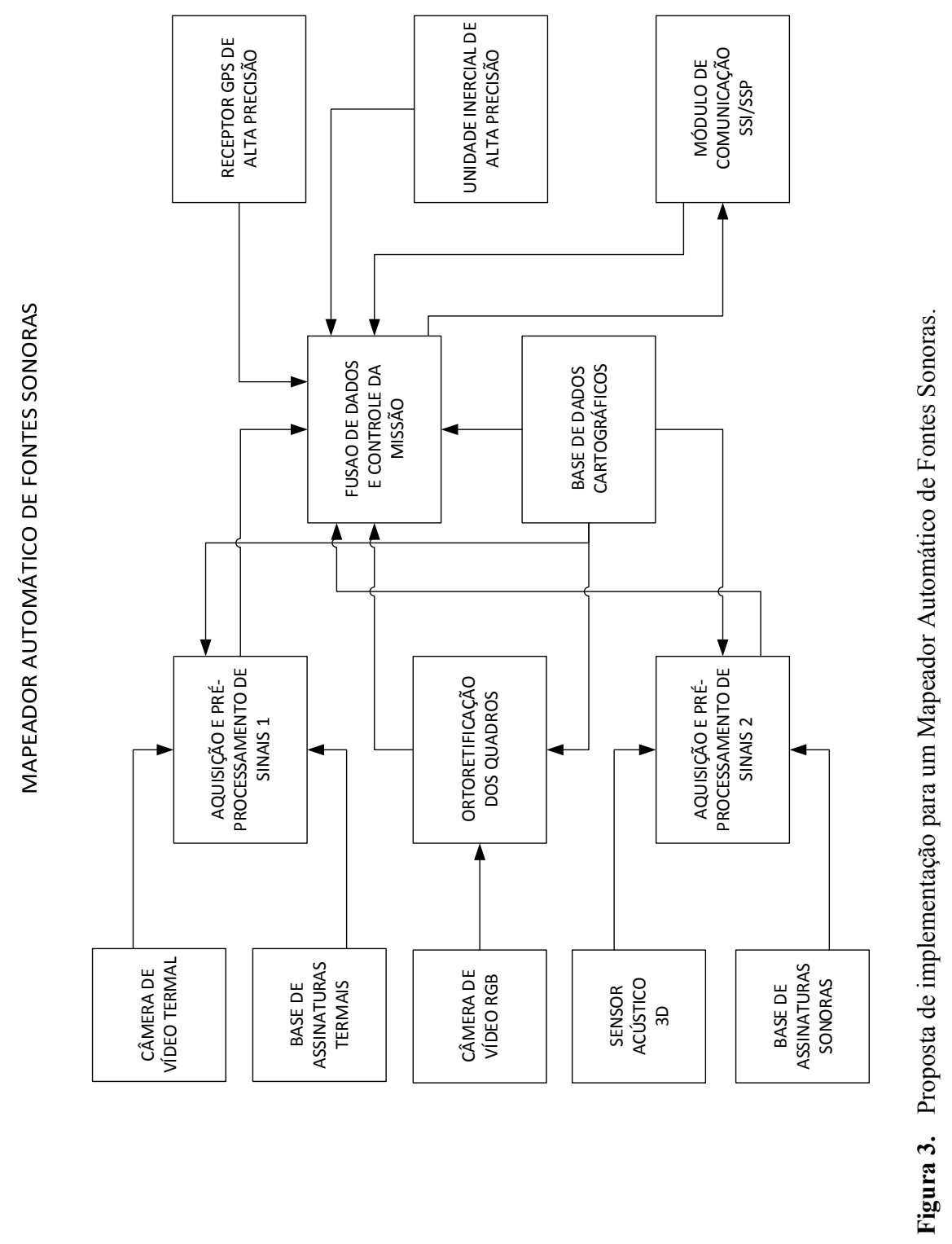


A modelagem ilustrada na Figura 3 foi proposta com base nas etapas de desenvolvimento de um MOSA (Figura 2), sendo composta pelos seguintes blocos:

- Sensores: câmera de vídeo termal, câmera de vídeo RGB, unidade inercial de alta precisão e receptor GPS de alta precisão;

- Bases de assinaturas termais: visam o reconhecimento automático de padrões/classificação de imagens térmicas;

- Base de assinaturas acústicas: visam o reconhecimento automático de padrões sonoros/classificação;

- Aquisição e pré-processamento de sinais 1: processamento básico das imagens termais incluindo correções radiométricas e geométricas;

- Aquisição e pré-processamento de sinais 2: processamento básico dos arquivos de áudio incluindo filtros de ruídos;

- Base de dados cartográficos: contém os documentos de referência necessários ao georreferenciamento direto aos demais processos;

- Ortoretificação dos quadros: corrige as distorções devido ao relevo através de modelos de elevação e outros documentos cartográficos de referência;

- Fusão de dados e controle da missão: integra os dados de diferentes sensores utilizando técnicas específicas, tais como filtros de Kalman e redes neurais artificiais. O controle da missão é capaz de alterar rotas em função das informações obtidas por meio da fusão de dados para que a missão possa ser adaptativa a situações atuais do cenário sobrevoado;

- Módulo de comunicação: realiza a conexão entre o MOSA e a aeronave (SSI/SSP).

\section{Conclusão e trabalhos futuros}

Este trabalho apresentou o conceito MOSA, sob um ponto de vista arquitetural, como um sistema integrado de informações para o sensoriamento remoto. O sistema incorpora hardware e software necessários para processar os dados brutos coletados por um conjunto de sensores que interagem entre si de maneira inteligente. A ideia principal é produzir em tempo real, informação geográfica e temática de pronto emprego, transmitida através de canais de comunicação de baixa largura de banda para uma estação de solo. Em solo, na ECS, um Sistema de Informação Geográfico poderia ser usado para produzir mapas temáticos derivados.

A arquitetura do MOSA é apresentada para destacar a integração de todos os conceitos envolvidos neste trabalho. A composição se dá através de mecanismos plug-and-play:SSP/SSI (Smart Sensor Protocol/Smart Sensor Intertace, permitindo troca de informação otimizada entre o MOSA e aeronave. Aeronave e MOSA comunicam-se durante a inicialização do sistema, trocando os requisitos da missão 
e parâmetros de voo (altitude, alcance, performance etc.), a fim de definir a viabilidade (total ou parcial) da missão.

São apresentadas e discutidas as etapas de desenvolvimento e a modelagem de referência de um MOSA concebido no contexto do monitoramento ambiental, no qual dados acústicos e ter mais são integrados para a obtenção de um mapa temático que reflita um quadro situacional inteligente da área de interesse sobrevoada.

Trabalhos futuros incluem testes de voo para validar aspectos de desempenho. Estes testes podem sugerir algumas mudanças no DFD proposto. Os primeiros resultados incentivam o desenvolvimento mostrando que o emprego de MOSA's pode representar um grande passo para uma melhor usabilidade de VANT's em sensoriamento remoto.

\section{Agradecimentos}

Os autores agradecem ao Instituto de Ciências Matemáticas e Computação da Universidade de São Paulo (ICMC-USP) e ao Exército Brasileiro.

\section{Bibliografia}

Abielmona, R.S. et al. (2010). "Distributed intelligent sensor agent system for environment mapping", Journal of Ambient Intelligence and Humanized Computing, Springer-Verlag, 1(2), 95-110.

Bitar, O.Y. e Ortega, R.D. (1998). "Gestão Ambiental”, in Oliveira, A.M.S., Brito, S.N.A. (Eds.), Geologia de Engenharia, São Paulo, Associação Brasileira de Geologia de Engenharia (ABGE), cap. 32, 499-508.

Braga et al. (2011). "Prolices: An approach to develop product lines for safetycritical embedded systems", in XXXVII Latin-American Informatic Conference (CLEI XXXVII), 1-16.

Choi, K e Lee, I. (2013). "A sequential aerial triangulation algorithm for real-time georeferencing of image sequences acquired by an airborne multi-sensor system", Remote Sensing, 5, 57-82.

GAO, U.S. Government Accountability Office (2008). "Unmanned aircraft systems - federal actions needed to ensure safety and expand their potential uses within the national airspace system", GAO-08-511.

Grigorius, C.; Dollas, A. e Bourbakis, N.G. (2012). "An embedded softwarereconfigurable color segmentation architecture for image processing systems", Microprocessors and Microsystems - Embedded Hardware Design, Elsevier Science Publishers B.V., Amsterdam, The Netherlands, 36(3), 215-231. 
Hruska, R.C. et al. (2005). "Small UAV-acquired, high-resolution, georeferenced still imagery", in AUVSI's Unmanned Systems North America, Proceedings... Idaho Nacional Laboratory (INL - US Energy Department), 837-840.

Kealy et al. (2013). "Collaborative navigation field trials with different sensor platforms", Workshop on Positioning Navigation and Communication (WPNC), 10th, Proceedings... IEEE, 1-6.

Klausner, A.; Rinner, B. and Tengg, A. (2006) "I-SENSE: Intelligent Embedded Multi- Sensor Fusion", in International Workshop on Intelligent Solutions in Embedded Systems, Vienna, Proceedings... IEEE, 1-12.

Li-Chee-Ming, J. and Armenakis, C. (2012). "Introduction to a portable stereo mapping system for unmanned vehicles", Geomatica, 66(4), 323-332.

Li Ying-cheng; Ye Dong-mei; Ding Xiao-bo; Teng Chang-sheng ; Wang Guang-hui and Li Tuan-hao (2011). "UAV Aerial Photography Technology in Island Topographic Mapping", Image and Data Fusion (ISIDF), International Symposium, 1-4.

Machado, P.A.L. (1995). "Direito ambiental brasileiro", 5a. ed. São Paulo, Malheiros Editores, 696 pp.

Mejías, L.; Mondragón, I.; Correa, J.F.; Campoy, P. (2007). “Colibri: Vision-guided helicopter for surveillance and visual inspection", in Video Proceedings of IEEE International Conference on Robotics and Automation, Proceedings... Rome, Italy, April, 2760-2761.

Molina, P. et al. (2012). "Searching lost people with UAV's: the system and results of the CLOSE-SEARCH project", in Congress of The International Society for Photogrammetry and Remote Sensing, 22nd, Melbourne, Australia, 2012. Proceedings... International Archives of the Photogrammetry, Remote Sensing and Spatial Information Sciences, 39(B1), 441-446.

Molina, P. et al. (2013); "Navigation and Remote Sensing payloads and methods of the SARVANT Unmanned Aerial System", Internacional Archieves of Photogrammetry, Remote Sensing and Spatial Information, XL-1-W2, 275-280.

Nagai et al. (2009). "UAV-Borne 3-D Mapping System by Multisensor Integration", IEEE Transactions on Geoscience and Remote Sensing Society, 47(3), 701-708.

OSD, Office of the Secretary of Defense (2002). "UAV Roadmap 2002-2027", (Acquisition, Technology, and Logistics) Air Warfare.

OSD, Office of the Secretary of Defense (2005). "Unmanned Aircraft Systems Roadmap 2005-2030”. (Acquisition, Technology, and Logistics) Air Warfare. 
OSD, Office of the Secretary of Defense (2009a). "Unmanned Systems Roadmap 2007-2032", (Acquisition, Technology, and Logistics) Air Warfare.

OSD, Office of the Secretary of Defense (2009b). "Unmanned Systems Integrated Roadmap FY2009-2034", (Acquisition, Technology, and Logistics) Air Warfare.

Polychronopoulos, A. et al. (2006). "Data fusion in multi sensor platforms for widearea perception", in Intelligent Vehicles Symposium, Proceedings... IEEE, 412 417.

Shaw, M., Garlan, D.; (1996). "Software Architecture. Perspectives on an Emerging", Discipline, Prentice Hall.

Trindade Jr., O., Neris, L.O., Barbosa, L., and Branco, K.R.L.J.C. (2010). “A Layered Approach to Design Autopilots", in IEEE-ICIT 2010 International Conference on Industrial Technology, Viña del Mar. IEEE-ICIT 2010 International Conference on Industrial Technology. Santiago, Chile: IEEE Press, vol. 1., 1395-1400.

Trindade Jr., O.; Barbosa, Neris, L.C.P.; L. O. and Jorge, L.A.C. (2002). “A Mission Planner and Navigation System for the ARARA Project". in ICAS 23rd International Congress of Aeronautical Sciences, Toronto.

Turolla, A.; Marchesotti, L. and Regazzoni, C.S. (2004). "Multicamera object tracking in video surveillance applications", in Target Tracking, Algorithms and Applications, IEE. Proceedings... IEEE, 85-90.

Ugolotti, R. et al.; (2013). "Multi-sensor system for detection and classification of human activities". Journal of Ambient Intelligence and Humanized Computing. Springer-Verlag, 4(1), 27-41.

USAF - United States Air Force Washington DC. (2009). "United States Air Force Unmanned Aircraft Systems Flight Plan 2009-2047”.

Valavanis, K.P. (2007). "Advances in Unmanned Aerial Vehicles: State of the Art and the Road to Autonomy", International Series on Intelligent Systems, Control, and Automation: Science and Engineering, vol. 33.

Yi Lin et al. (2013). "Development of a UAV-MMS-Collaborative Aerial-toGround Remote Sensing System - A Preparatory Field Validation". Journal of Selected Topics in Applied Earth Observations and Remote Sensing. IEEE, 6(4), 1893-1898.

Zhang C. and Kovacs, J.M. (2012). "The application of small unmanned aerial systems for precision agriculture: A review", Precision Agriculture, 13(6), 693712 . 